\title{
Application of Wavelet Transform for Image Denoising of Spatially and Time Variable Astronomical Imaging Systems
}

\author{
M. Blažek, E. Anisimova, P. Páta
}

\begin{abstract}
We report on our efforts to formulate algorithms for image signal processing with the spatially and time variant PointSpread Function (PSF) and inhomogeneous noise of real imaging systems. In this paper we focus on application of the wavelet transform for denoising of the astronomical images with complicated conditions. They influence above all accuracy of the measurements and the new source detection ability. Our aim is to test the usefulness of Wavelet transform (as the standard image processing technique) for astronomical purposes.
\end{abstract}

Keywords: Point-Spread Function, image processing, IRAF, Daophot, wide-field, noise reduction, wavelet, astronomy.

\section{Introduction}

Our research reflects the needs for effective data processing from systems with complicated PSF or important role of noise like wide-field cameras or precise astronomical telescopes (e.g. MAIA [1], BART, D50 [2] and BOOTES [3]). A large amount of data is displaced because of big errors during data reduction (e.g. information found on the edges of the image from fish-eye type cameras).

Standard photometrical packages (e.g. IRAF [4]) include algorithms for PSF extraction dependent linearly or quadratically on the position in the image [5]. However, this does not change the analytic part of PSF - only the fitting residua occurring in the lookup table (the experimental part of the constructed PSF) depending on the image cursor, where the convolution proceeds. This model however does not reshape the stars at the edges of wide-field images (an example of such an asymmetric PSF is shown in Figure 1), although it is useful in the crowded fields as shown in Figure 2.

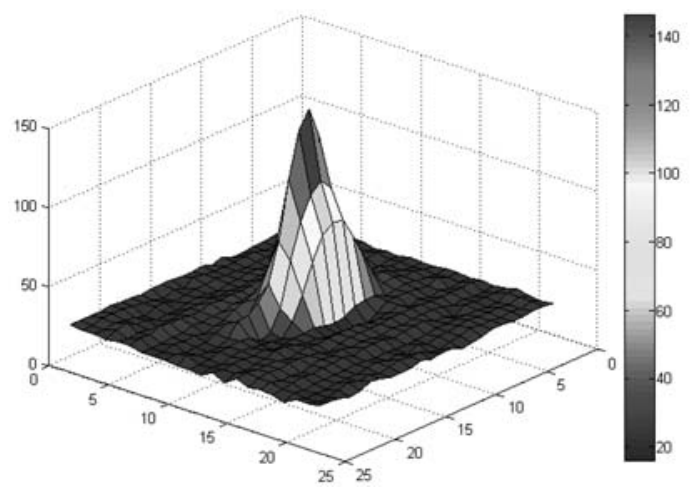

Fig. 1: Example of the asymmetric stellar Point-Spread Function from the edge of a wide-field camera
The formulation of new noise space dependent models (other than simple darkframe) and automatic variant PSF construction is above all useful for widefield detectors. With the new algorithms, higher precision (sensitivity) and lower data littering can be obtained. All-sky cameras for Gamma-Ray Bursts or meteor detections require optoelectronic analysis specific for each system because of the different dependence of the parameters.

Automatic data reduction usually does not include time variant PSF because of the slow changes of the external conditions (camera cooling, atmosphere, direction of observation), which become important during long and precise measurement. The parameters of PSF photometry (in comparison to aperture photometry) have to be acquired manually. The algorithms for evaluating them automatically on series of images will enable faster and more precise photometry if we assume similar and slowly changing conditions of the observations. Typically one-by-one image analysis does not expect the dependence of the PSF parameters on the previous images.

Aberration modelling using Zernike polynomials [6] can provide better results for space-variant systems, while the Fourier or Wavelet transform can provide guidance for time-variant analysis of PSF.

\section{Wavelet transform for astronomic image processing}

The discrete wavelet transform [7] is widely used in image processing to get rid of noise, while it is not commonly used in astronomy as higher uncertainties can occur. We tested the application of this method on stellar field data taken by the robotic tele- 

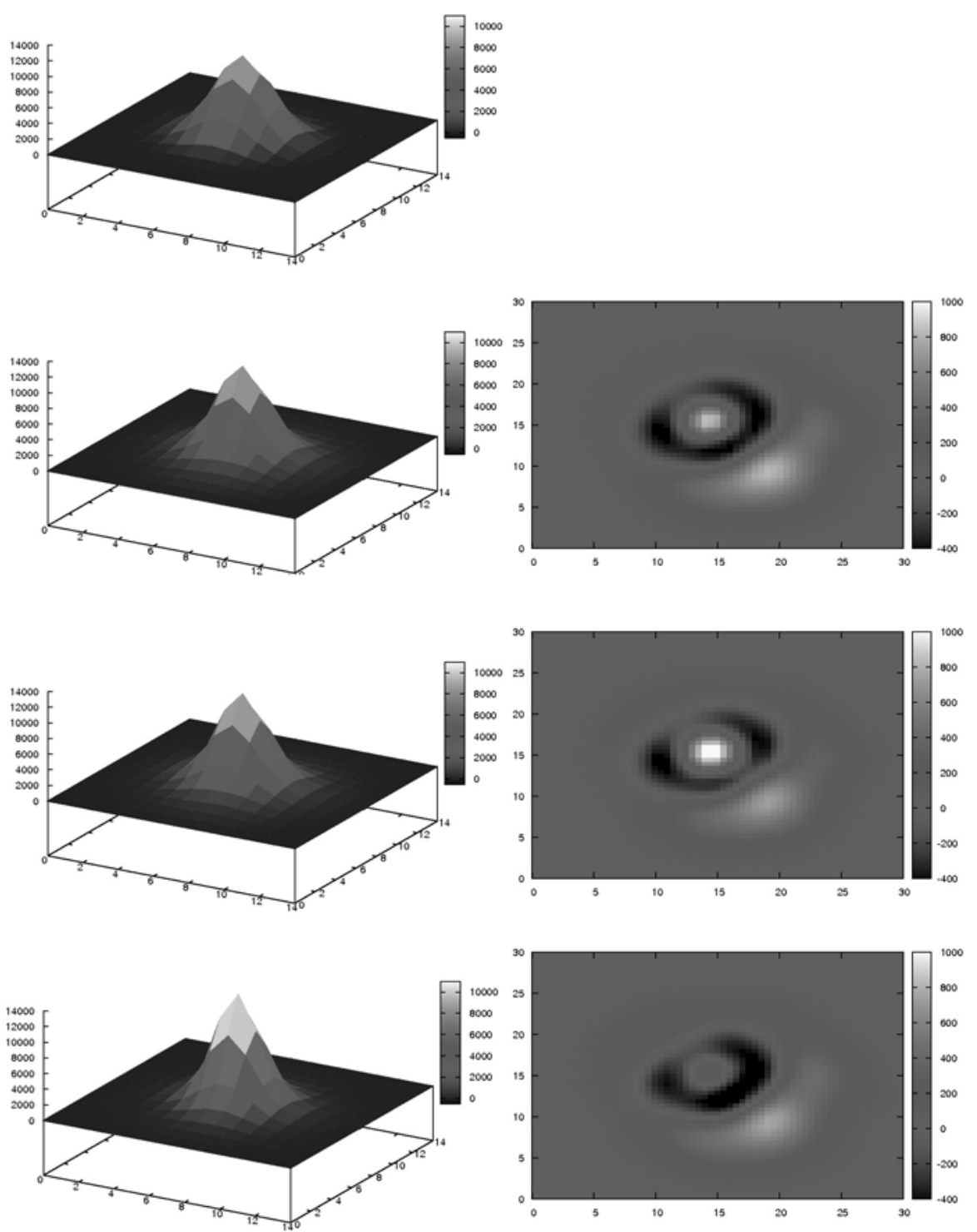

Fig. 2: Methods of PSF construction from an over-crowded stellar field (NGC 6791) — significant coma present. The second column represents look-up tables - additive corrections from the analytic function to the observed empirical PSF [5]

I. row - analytic function only

II. row - constant PSF over the image containing both the analytic function and corrections

III. row - look-up tables depending linearly on position

IV. row - look-up tables depending quadratically on position

scope 'D50' at the Ondrejov observatory (CZ). The wavelet transform was proceeded with Matlab ${ }^{1}$ software, while IRAF software was used for astronomic processing with the DAOPHOT package ${ }^{2}[5]$.

We focused on 3 specific targets:

- denoising

- stellar magnitude changes

- the influence of the wavelet transform on the efficiency of the new object detection algorithms
Five specific wavelet families were used for the tests: Daubechies, Biorthogonal, Reverse Biorthogonal, Symlets and Coiflets, with the decomposition level up to 3 [8]. Those families were chosen to cover both orthogonal and biorthogonal wavelet shapes and the most used wavelet families in standard image signal processing. Only soft thresholding [9] was applied, due to its better peak signal to noise ratio for image denoising, and no thresholding was done to

\footnotetext{
${ }^{1}$ http://www.mathworks.com/

${ }^{2}$ http://iraf.noao.edu/
} 
the approximation coefficients of the wavelet dyadic decomposition. The test image is shown in Figure 3. The efficiency of the wavelet transform on the test image for each wavelet was checked using the DAOPHOT routines [5] under IRAF software package and with standard aperture photometry algorithms.

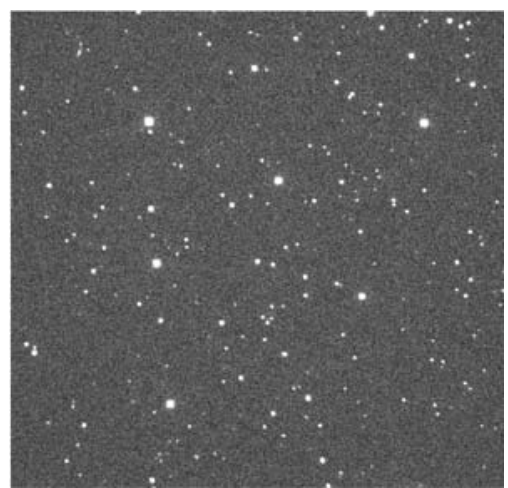

Fig. 3: Test image for wavelet transform

\subsection{Results of the denoising}

We observed the changes of the standard deviation of the sky (background) value depending strongly on the decomposition level and the intensities of the nearby stars. The first level of decomposition shows only small variations among the wavelets that were used, lowering the noise dispersion by $40 \%-50 \%$. Even greater efficiency can be gained around faint stars for the second decomposition level with higher differences in the influence between the wavelets. For the third decomposition level, the influence of the denoising on the astronomical image was even negative for several wavelets (e.g. most of the Daubechies and Reversed Biorthogonal wavelets) because of the strong artifacts of the transform (Figure 4). All wavelet denoising results can be seen in Table 1 . The first and second column of the table represent the wavelet that was used. The first column for each decomposition level shows the mean percentage lower- ing (effectivity $E$ ) of the background noise deviation $\sigma$ around stars according to the equation

$$
E=\frac{1-\sigma_{A}}{\sigma_{B}}
$$

where the subscripts $B$ and $A$ represent background noise deviation before the wavelet transform application and after the transform respectively. The second and third column for each decomposition level in Table 1 show the maximum $\Delta m_{\max }$ and mean $\overline{\Delta m}$ errors of the photometric magnitudes after the wavelet transform. The Signal-To-Noise Ratio of the stars selected for the statistics is from 150 to 3100 .

\subsection{Magnitude changes}

Most of the photometrical magnitudes were unchanged or changed only slightly during the wavelet transform, and all of those changes were hidden inside the 3- $\sigma$ band of the counted instrumental magnitudes. From this point of view, the wavelet transform can therefore be considered as a low-loss modification of scientific images with low influence on the photometrical information. Detailed errors of the magnitude changes can be seen in Table 1 .

\subsection{Detection of new sources}

The same detection algorithms for transformed images as for the original images were used for the search of new object candidates and for the pairing with their counterparts in the astronomical catalogues. However, no new sources were found using the DAOPHOT routines [5]. The influence of the wavelets on the Point-Spread Function of the image was unfortunately too strong to get any new information from the noise level. No advantages for the new source detection algorithms were therefore obtained from the wavelet denoising. Future research is therefore suggested.

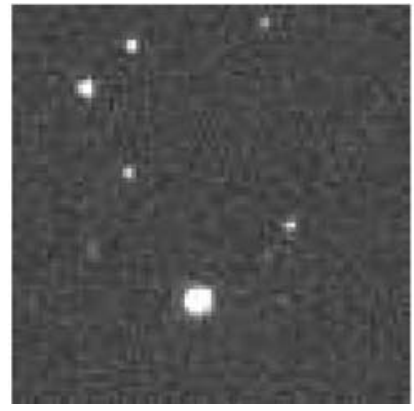

(a)

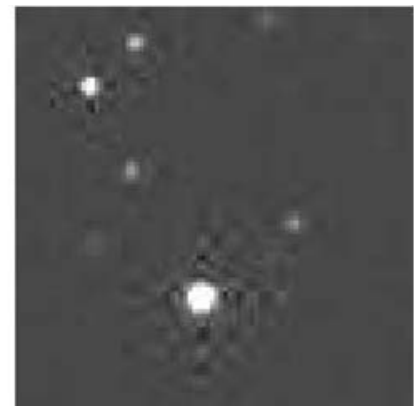

(b)

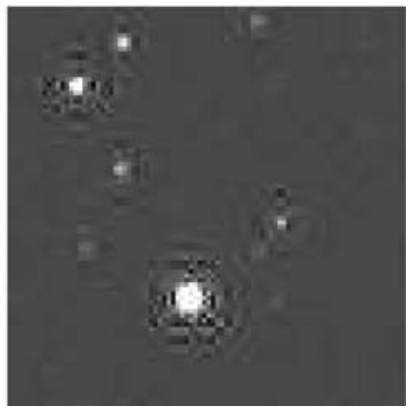

(c)

Fig. 4: Effect of the wavelet transform on (a) the original stellar field,

(b) the Daubechies 3 wavelet third decomposition level,

(c) the Reverse Biorthogonal 3.3 wavelet third decomposition level 
Table 1: Efficiency $E$ of the denoising of the background deviation of the stellar surroundings (1). Maximum $\Delta m_{\max }$ and mean $\overline{\Delta m}$ error of photometry after application of given Wavelet transform

\begin{tabular}{|c|c|c|c|c|c|c|c|c|c|c|}
\hline \multicolumn{2}{|l|}{ Wavelet } & \multicolumn{3}{|c|}{ Dec. Level 1} & \multicolumn{3}{|c|}{ Dec. Level 2} & \multicolumn{3}{|c|}{ Dec. Level 3} \\
\hline & & $\begin{array}{c}E \\
{[\%]} \\
\end{array}$ & $\begin{array}{c}\Delta m_{\max } \\
{[\mathrm{mag}]} \\
\end{array}$ & $\begin{array}{c}\overline{\Delta m} \\
{[\mathrm{mag}]}\end{array}$ & $\begin{array}{c}E \\
{[\%]} \\
\end{array}$ & $\begin{array}{c}\Delta m_{\max } \\
{[\mathrm{mag}]}\end{array}$ & $\begin{array}{c}\overline{\Delta m} \\
{[\mathrm{mag}]}\end{array}$ & $\begin{array}{r}E \\
{[\%]} \\
\end{array}$ & $\begin{array}{c}\Delta m_{\max } \\
{[\mathrm{mag}]}\end{array}$ & $\begin{array}{c}\overline{\Delta m} \\
{[\mathrm{mag}]}\end{array}$ \\
\hline \multirow[t]{10}{*}{ Daubechies } & 1 & 47 & 0.026 & 0.010 & 71 & 0.052 & 0.020 & 79 & 0.098 & 0.031 \\
\hline & 2 & 47 & 0.014 & 0.004 & 73 & 0.030 & 0.012 & 55 & 0.067 & 0.026 \\
\hline & 3 & 47 & 0.005 & 0.002 & 69 & 0.046 & 0.014 & 35 & 0.102 & 0.030 \\
\hline & 4 & 48 & 0.008 & 0.002 & 60 & 0.037 & 0.010 & 24 & 0.092 & 0.029 \\
\hline & 5 & 48 & 0.012 & 0.002 & 57 & 0.020 & 0.006 & 22 & 0.061 & 0.026 \\
\hline & 6 & 48 & 0.012 & 0.003 & 43 & 0.021 & 0.008 & 1 & 0.094 & 0.034 \\
\hline & 7 & 46 & 0.011 & 0.003 & 39 & 0.039 & 0.007 & 4 & 0.114 & 0.032 \\
\hline & 8 & 44 & 0.008 & 0.002 & 49 & 0.013 & 0.005 & 10 & 0.090 & 0.034 \\
\hline & 9 & 45 & 0.008 & 0.002 & 34 & 0.021 & 0.007 & -7 & 0.118 & 0.037 \\
\hline & 10 & 45 & 0.014 & 0.002 & 28 & 0.036 & 0.008 & -10 & 0.122 & 0.039 \\
\hline \multirow[t]{15}{*}{ Biorthogonal } & 1.1 & 47 & 0.026 & 0.010 & 71 & 0.052 & 0.020 & 79 & 0.098 & 0.031 \\
\hline & 1.3 & 45 & 0.007 & 0.002 & 72 & 0.017 & 0.006 & 58 & 0.061 & 0.016 \\
\hline & 1.5 & 43 & 0.004 & 0.001 & 70 & 0.012 & 0.002 & 51 & 0.044 & 0.012 \\
\hline & 2.2 & 48 & 0.010 & 0.004 & 69 & 0.060 & 0.016 & 71 & 0.110 & 0.023 \\
\hline & 2.4 & 48 & 0.006 & 0.001 & 70 & 0.025 & 0.009 & 66 & 0.051 & 0.017 \\
\hline & 2.6 & 48 & 0.008 & 0.001 & 69 & 0.032 & 0.007 & 54 & 0.055 & 0.016 \\
\hline & 2.8 & 48 & 0.009 & 0.002 & 71 & 0.014 & 0.004 & 56 & 0.040 & 0.012 \\
\hline & 3.1 & 34 & 0.012 & 0.005 & 45 & 0.076 & 0.016 & 38 & 0.102 & 0.018 \\
\hline & 3.3 & 45 & 0.008 & 0.002 & 67 & 0.025 & 0.007 & 58 & 0.028 & 0.008 \\
\hline & 3.5 & 48 & 0.010 & 0.002 & 67 & 0.037 & 0.008 & 53 & 0.070 & 0.012 \\
\hline & 3.7 & 48 & 0.011 & 0.002 & 68 & 0.015 & 0.005 & - & - & - \\
\hline & 3.9 & 48 & 0.012 & 0.002 & 61 & 0.018 & 0.005 & 47 & 0.033 & 0.009 \\
\hline & 4.4 & 48 & 0.012 & 0.002 & 68 & 0.025 & 0.009 & 37 & 0.077 & 0.026 \\
\hline & 5.5 & 44 & 0.009 & 0.001 & 64 & 0.018 & 0.006 & -15 & 0.080 & 0.026 \\
\hline & 6.8 & 47 & 0.014 & 0.003 & 65 & 0.018 & 0.006 & 11 & 0.064 & 0.024 \\
\hline \multirow[t]{5}{*}{ Coiflets } & 1 & 48 & 0.010 & 0.003 & 71 & 0.053 & 0.014 & 61 & 0.099 & 0.026 \\
\hline & 2 & 48 & 0.010 & 0.002 & 66 & 0.043 & 0.009 & 26 & 0.083 & 0.027 \\
\hline & 3 & 47 & 0.013 & 0.003 & 55 & 0.039 & 0.007 & 15 & 0.099 & 0.025 \\
\hline & 4 & 47 & 0.014 & 0.003 & 42 & 0.041 & 0.007 & 1 & 0.090 & 0.028 \\
\hline & 5 & 47 & 0.014 & 0.003 & 30 & 0.041 & 0.007 & -10 & 0.105 & 0.027 \\
\hline \multirow[t]{7}{*}{ Symlets } & 2 & 47 & 0.014 & 0.004 & 73 & 0.030 & 0.012 & 55 & 0.067 & 0.026 \\
\hline & 3 & 47 & 0.005 & 0.002 & 69 & 0.046 & 0.014 & 35 & 0.102 & 0.030 \\
\hline & 4 & 47 & 0.008 & 0.002 & 66 & 0.046 & 0.011 & 31 & 0.086 & 0.026 \\
\hline & 5 & 45 & 0.007 & 0.002 & 62 & 0.045 & 0.010 & 23 & 0.119 & 0.028 \\
\hline & 6 & 47 & 0.011 & 0.003 & 61 & 0.015 & 0.005 & 18 & 0.060 & 0.023 \\
\hline & 7 & 46 & 0.015 & 0.002 & 51 & 0.165 & 0.009 & - & 0.068 & 0.028 \\
\hline & 8 & 47 & 0.012 & 0.003 & 43 & 0.045 & 0.008 & 2 & 0.099 & 0.029 \\
\hline Reverse & 1.1 & 47 & 0.026 & 0.010 & 71 & 0.052 & 0.020 & 79 & 0.098 & 0.031 \\
\hline \multirow[t]{13}{*}{ Biorthogonal } & 1.3 & 44 & 0.015 & 0.004 & 68 & 0.054 & 0.016 & 45 & 0.118 & 0.031 \\
\hline & 1.5 & 43 & 0.006 & 0.002 & 65 & 0.034 & 0.007 & 23 & 0.106 & 0.021 \\
\hline & 2.2 & 51 & 0.009 & 0.002 & 66 & 0.044 & 0.010 & 28 & 0.087 & 0.020 \\
\hline & 2.4 & 49 & 0.007 & 0.002 & 67 & 0.018 & 0.007 & 11 & 0.057 & 0.020 \\
\hline & 2.6 & 47 & 0.010 & 0.002 & 57 & 0.035 & 0.008 & 14 & 0.067 & 0.022 \\
\hline & 2.8 & 47 & 0.011 & 0.002 & 47 & 0.017 & 0.005 & 8 & 0.063 & 0.019 \\
\hline & 3.1 & 34 & 0.006 & 0.002 & 11 & 0.019 & 0.004 & -115 & 0.039 & 0.009 \\
\hline & 3.3 & 45 & 0.004 & 0.002 & 32 & 0.015 & 0.004 & -85 & 0.030 & 0.010 \\
\hline & 3.5 & 47 & 0.006 & 0.002 & 36 & 0.020 & 0.006 & -53 & 0.057 & 0.014 \\
\hline & 3.7 & 48 & 0.009 & 0.002 & 33 & 0.015 & 0.005 & -9 & 0.043 & 0.011 \\
\hline & 3.9 & 48 & 0.010 & 0.002 & 28 & 0.019 & 0.006 & -9 & 0.055 & 0.016 \\
\hline & 4.4 & 48 & 0.009 & 0.002 & 67 & 0.015 & 0.006 & 24 & 0.046 & 0.019 \\
\hline & 5.5 & 48 & 0.013 & 0.003 & 64 & 0.010 & 0.003 & 12 & 0.063 & 0.023 \\
\hline
\end{tabular}




\section{Conclusion}

Space dependent PSF and noise models are necessary for precise and complete data reduction from imaging systems with wide-field lenses. We therefore plan to measure the optoelectronic characteristics and describe the geometric distortion of all-sky detectors to test and formulate new algorithms of variant noise and PSF. The advantage of the denoising algorithms should be namely new discoveries of objects hidden in the noise. Although the background noise distribution was positively modified with most of the wavelet transforms, no advantage for the new source detection algorithms was found yet. In our future effort we'd like to focus as well on the detection algorithms of the transformed astronomical images to obtain photometric methods possibly useful in practice.

\section{Acknowledgement}

This project was supported by the Czech Technical University in Prague grant SGS CVUT 2010 OHK3-066/10.

\section{References}

[1] Vítek, S., Koten, P., Páta, P., Fliegel, K.: Double-station automatic video observation of the meteors, Advances in Astronomy 2010, Article ID 943145, 4 pages (2010).

[2] Nekola, M. et al: Robotic telescopes for high energy astrophysics in Ondřejov, Experimental Astronomy, Vol. 28, 2010, Issue 1, p. 79-85.

[3] Castro-Tirado, A. J. et al.: The Burst Observer and Optical Transient Exploring Sys- tem (BOOTES), A\&A S Suppl., Vol. 138, 1999, p. $583-585$.

[4] Massey, P.: A User's Guide to CCD Reductions with IRAF, NOAO (1997).

[5] Stetson, Peter B.: DAOPHOT - A computer program for crowded-field stellar photometry, Astronomical Society of the Pacific, Publications, Vol. 99, 1987, p. 191-222.

[6] Řerábek, M., Páta, P., Koten, P.: Processing of the astronomical image data obtained from uwfc optical systems, Image Reconstruction from Incomplete Data $V$, Proc. SPIE 7076, 70760L (2008).

[7] Mallat, S.: A theory for multiresolution signal decomposition: the wavelet representation, IEEE Pattern Anal. and Machine Intell., Vol. 11, no. 7, 1989, pp. 674-693.

[8] Daubechies, I.: Ten lectures on wavelets, CBMSNSF regional conference series in applied mathematics, Lectures delivered at the CBMS conference on wavelets, University of Lowell, Mass., June 1990, Philadelphia: Society for Industrial and Applied Mathematics - SIAM (1992).

[9] Donoho, D. L.: De-noising by soft-thresholding, IEEE, Trans. on Inf. Theory, Vol. 41, 3, 1995, pp. 613-627.

Martin Blažek

E-mail: blazem10@fel.cvut.cz

Elena Anisimova

Petr Páta

Czech Technical University in Prague

Faculty of Electrical Engineering

Technická 2, Prague 6 